\title{
Sur un critère de Báez-Duarte pour l'hypothèse de Riemann
}

\author{
Michel Balazard et Anne de Roton
}

8 novembre 2018

Pour Luis Báez-Duarte, à l'occasion de son soixante-dixième anniversaire.

Abstract

\begin{abstract}
Define $e_{n}(t)=\{t / n\}$. Let $d_{N}$ denote the distance in $L^{2}\left(0, \infty ; t^{-2} d t\right)$ between the indicator function of [1, $\infty$ [ and the vector space generated by $e_{1}, \ldots, e_{N}$. A theorem of Báez-Duarte states that the Riemann hypothesis (RH) holds if and only if $d_{N} \rightarrow 0$ when $N \rightarrow \infty$. Assuming RH, we prove the estimate

$$
d_{N}^{2} \leqslant(\log \log N)^{5 / 2+o(1)}(\log N)^{-1 / 2} .
$$
\end{abstract}

\section{KEYWORDS}

Riemann zeta function, Riemann hypothesis, Báez-Duarte criterion, Möbius function. MSC classification : $11 \mathrm{M} 26$

\section{Position du problème et énoncé du résultat principal}

L'étude de la répartition des nombres premiers se ramène à la recherche d'approximations de la fonction

$$
\chi(x)=[x \geqslant 1]
$$

par des combinaisons linéaires

$$
\varphi(x)=\sum_{n=1}^{N} c_{n}\{x / n\} \quad\left(N \in \mathbb{N}, c_{n} \in \mathbb{R}\right)
$$

de dilatées de la fonction «partie fractionnaire». Ce fait est connu depuis Tchebychev (cf. [15]). En choisissant

$$
\varphi(x)=-\{x\}+\{x / 2\}+\{x / 3\}+\{x / 5\}-\{x / 30\}
$$

il avait observé l'encadrement

$$
\varphi(x) \leqslant \chi(x) \leqslant \sum_{k \geqslant 0} \varphi\left(x / 6^{k}\right)
$$

pour en déduire

$$
A x+O(\log x) \leqslant \sum_{n \leqslant x} \Lambda(n) \leqslant \frac{6}{5} A x+O\left(\log ^{2} x\right)
$$


où $\Lambda$ désigne la fonction de von Mangoldt, et

$$
A=\log \frac{2^{1 / 2} 3^{1 / 3} 5^{1 / 5}}{30^{1 / 30}}=0,92129202 \ldots .
$$

On peut préciser la nature de l'approximation de (1) par (2) équivalente au théorème des nombres premiers

$$
\sum_{n \leqslant x} \Lambda(n) \sim x \quad(x \rightarrow \infty),
$$

où à l'hypothèse de Riemann

$$
\sum_{n \leqslant x} \Lambda(n)=x+O_{\delta}\left(x^{\frac{1}{2}+\delta}\right) \quad(x \geqslant 1, \delta>0) .
$$

Ainsi, le théorème des nombres premiers est équivalent国à l'assertion

$$
\inf _{\varphi} \int_{0}^{\infty}|\chi(x)-\varphi(x)| \frac{d x}{x^{2}}=0 .
$$

Quant à l'hypothèse de Riemann, Báez-Duarte (cf. 4]) a démontré qu'elle équivaut à

$$
\inf _{\varphi} \int_{0}^{\infty}|\chi(x)-\varphi(x)|^{2} \frac{d x}{x^{2}}=0 .
$$

Dans les deux cas, l'infimum est pris sur les $\varphi$ de la forme (2).

Nous nous intéressons dans cet article à une forme quantitative de ce critère. Soit $H$ l'espace de Hilbert $L^{2}\left(0, \infty ; t^{-2} d t\right)$ et, pour $\alpha>0$,

$$
e_{\alpha}(t)=\{t / \alpha\} \quad(t>0)
$$

Posons, pour $N$ entier positif,

$$
d_{N}=\operatorname{dist}_{H}\left(\chi, \operatorname{Vect}\left(e_{1}, \ldots, e_{N}\right)\right) .
$$

Ainsi, le critère de Báez-Duarte affirme que l'hypothèse de Riemann équivaut à la convergence de $d_{N}$ vers 0 , quand $N$ tend vers l'infini.

Examinons maintenant la vitesse de cette convergence. D'une part, Burnol (cf. [6]) a démontré que

$$
d_{N}^{2} \geqslant \frac{C+o(1)}{\log N}, \quad N \rightarrow+\infty,
$$

où

$$
C=\sum_{\rho} \frac{\mathrm{m}(\rho)^{2}}{|\rho|^{2}}
$$

la somme portant sur les zéros non triviaux $\rho$ de la fonction $\zeta$, et $\mathrm{m}(\rho)$ désignant la multiplicité de $\rho$ comme zéro de $\zeta$.

\footnotetext{
*Bien entendu, deux énoncés vrais sont toujours équivalents; nous renvoyons à [9] et [1] pour des énoncés précis sur ce sujet.
} 
Comme

$$
\sum_{\rho} \frac{\mathrm{m}(\rho)}{|\rho|^{2}}=2+\gamma-\log (4 \pi)
$$

(si l'hypothèse de Riemann est vraie, cf. [8], chapter $12,(10),(11)$ ), on en déduit en particulier que

$$
d_{N}^{2} \geqslant \frac{2+\gamma-\log (4 \pi)+o(1)}{\log N}, \quad N \rightarrow+\infty .
$$

D'autre part, les auteurs de 3] conjecturent l'égalité dans (3). Cette conjecture entraîne donc l'hypothèse de Riemann et la simplicité des zéros de $\zeta$.

Le comportement asymptotique de $d_{N}$ est difficile à déterminer, même conditionnellement à l'hypothèse de Riemann et d'autres conjectures classiques (simplicité des zéros de $\zeta$, conjecture de Montgomery sur la corrélation par paires,...). Dans 4, Báez-Duarte donne une démonstration (dûe au premier auteur) de la majoration

$$
d_{N}^{2} \ll(\log \log N)^{-2 / 3}
$$

sous l'hypothèse de Riemann. Nous améliorons ce résultat dans le présent travail.

Théorème L'hypothèse de Riemann entraîne que

$$
d_{N}^{2} \ll_{\delta}(\log \log N)^{5 / 2+\delta}(\log N)^{-1 / 2} \quad(N \geqslant 3),
$$

pour tout $\delta>0$.

Le plan de notre article est le suivant. Au §2 nous rappelons le rôle de la fonction de Möbius dans ce problème. Nous y majorons $d_{N}^{2}$ par la somme de deux quantités, $I_{N, \varepsilon}$ et $J_{\varepsilon}$, où $\varepsilon$ est un paramètre positif, et nous énonçons les estimations de ces quantités qui permettent de démontrer notre théorème. Le $₫ 3$ contient une étude de la fonction $\zeta(s) / \zeta(s+\varepsilon)$ nécessaire à la majoration, au $\varangle$ de la quantité $J_{\varepsilon}$. Les $\S \$ 5$ et 6 concernent l'estimation des sommes partielles de la série de Dirichlet de l'inverse de la fonction $\zeta$. Cela nous permet de majorer $I_{N, \varepsilon}$ au $\$ 7$. concluant ainsi la démonstration.

Il apparaîtra clairement que notre travail doit beaucoup à l'article récent [14. Nous remercions son auteur, Kannan Soundararajan, pour une correspondance instructive concernant [14].

Le paramètre $\delta$ est fixé une fois pour toutes. On suppose $0<\delta \leqslant 1 / 2$. On pose pour tout nombre complexe $s$

$$
\sigma=\Re s, \quad \tau=\Im s .
$$

Les symboles de Bachmann $O$ et de Vinogradov $\ll\left(\right.$ resp. $\left.\ll_{\delta}\right)$ qui apparaissent sous-entendent toujours des constantes absolues (resp. dépendant uniquement de $\delta$ ) et effectivement calculables. Enfin nous indiquerons, par les initiales $(H R)$ placées au début de l'énoncé d'une proposition, que la démonstration que nous en donnons utilise l'hypothèse de Riemann.

\section{Pertinence de la fonction de Möbius}

Partant de l'identité

$$
\chi=-\sum_{n \geqslant 1} \mu(n) e_{n}
$$


(valable au sens de la convergence simple), Báez-Duarte a d'abord montré (cf. [2]) la divergence dans $H$ de la série du second membre. Il a ensuite proposé d'approcher $\chi$ dans $H$ par les sommes

$$
-\sum_{n \leqslant N} \mu(n) n^{-\varepsilon} e_{n}
$$

où $\varepsilon$ est un paramètre positif, à choisir convenablement en fonction de $N$.

En posant

$$
\nu_{N, \varepsilon}=\left\|\chi+\sum_{n \leqslant N} \mu(n) n^{-\varepsilon} e_{n}\right\|_{H}^{2},
$$

on a évidemment $d_{N}^{2} \leqslant \nu_{N, \varepsilon}$ pour $N \geqslant 1, \varepsilon>0$. Posons maintenant pour $N \geqslant 1$ et $s \in \mathbb{C}$ :

$$
M_{N}(s)=\sum_{n \leqslant N} \mu(n) n^{-s} .
$$

On sait depuis Littlewood (cf. 11]) que l'hypothèse de Riemann entraîne la convergence de $M_{N}(s)$ vers $\frac{1}{\zeta(s)}$ quand $N$ tend vers l'infini, pour tout $s$ tel que $\Re s>\frac{1}{2}$. Nous allons faire apparaître la différence $M_{N}-1 / \zeta$ pour majorer $\nu_{N, \varepsilon}$.

Proposition 1 Pour $N \geqslant 1$ et $\varepsilon>0$, on a

$$
\nu_{N, \varepsilon} \leqslant 2 I_{N, \varepsilon}+2 J_{\varepsilon}
$$

où

$$
I_{N, \varepsilon}=\frac{1}{2 \pi} \int_{\sigma=1 / 2}|\zeta(s)|^{2}\left|M_{N}(s+\varepsilon)-\zeta(s+\varepsilon)^{-1}\right|^{2} \frac{d \tau}{|s|^{2}} \quad \text { et } \quad J_{\varepsilon}=\frac{1}{2 \pi} \int_{\sigma=1 / 2}\left|\frac{\zeta(s)}{\zeta(s+\varepsilon)}-1\right|^{2} \frac{d \tau}{|s|^{2}}
$$

\section{Démonstration}

La transformation de Mellin associe à toute fonction $f \in H$ une fonction $\mathfrak{M} f$, définie pour presque tout $s$ tel que $\sigma=1 / 2$ par la formule

$$
\mathfrak{M} f(s)=\int_{0}^{+\infty} f(t) t^{-s-1} d t
$$

(où $\int_{0}^{+\infty}$ signifie $\lim _{T \rightarrow+\infty} \int_{1 / T}^{T}$ ).

De plus, le théorème de Plancherel affirme que $f \mapsto \mathfrak{M} f$ est un opérateur unitaire entre $H$ et $L^{2}\left(\frac{1}{2}+i \mathbb{R}, d \tau / 2 \pi\right)$, espace que nous noterons simplement $L^{2}$. Comme

$$
\mathfrak{M} e_{\alpha}(s)=\alpha^{-s} \frac{\zeta(s)}{-s}, \quad \mathfrak{M} \chi(s)=\frac{1}{s}
$$


on a

$$
\begin{aligned}
\nu_{N, \varepsilon} & =\left\|\chi+\sum_{n \leqslant N} \mu(n) n^{-\varepsilon} e_{n}\right\|_{H}^{2} \\
& =\left\|\frac{1}{s}+\sum_{n \leqslant N} \mu(n) n^{-\varepsilon} n^{-s} \frac{\zeta(s)}{-s}\right\|_{L^{2}}^{2} \\
& =\frac{1}{2 \pi} \int_{\sigma=1 / 2}\left|1-\zeta(s) M_{N}(s+\varepsilon)\right|^{2} \frac{d \tau}{|s|^{2}} \\
& \leqslant \frac{1}{\pi} \int_{\sigma=1 / 2}\left|1-\frac{\zeta(s)}{\zeta(s+\varepsilon)}\right|^{2} \frac{d \tau}{|s|^{2}}+\frac{1}{\pi} \int_{\sigma=1 / 2}\left|\frac{\zeta(s)}{\zeta(s+\varepsilon)}-\zeta(s) M_{N}(s+\varepsilon)\right|^{2} \frac{d \tau}{|s|^{2}} \\
& \text { (où l'on a utilisé l'inégalité } \left.|a+b|^{2} \leqslant 2\left(|a|^{2}+|b|^{2}\right)\right) \\
& =2 J_{\varepsilon}+2 I_{N, \varepsilon} .
\end{aligned}
$$

Observons que la proposition 1 ne dépend pas de l'hypothèse de Riemann, mais que les quantités $I_{N, \varepsilon}$ et $J_{\varepsilon}$ pourraient être infinies si elle était fausse.

Dans 4], Báez-Duarte démontre (sous l'hypothèse de Riemann) que $I_{N, \varepsilon}$ tend vers 0 quand $N$ tend vers l'infini (pour tout $\varepsilon>0$ fixé), et que $J(\varepsilon)$ tend vers 0 quand $\varepsilon$ tend vers 0 . On a donc bien $d_{N}=o(1)$.

La version quantitative donnée dans [4] repose sur les estimations

$$
J(\varepsilon) \ll \varepsilon^{2 / 3} \quad(0<\varepsilon \leqslant 1 / 2),
$$

et

$$
I_{N, \varepsilon} \ll N^{-2 \varepsilon / 3} \quad(c / \log \log N \leqslant \varepsilon \leqslant 1 / 2),
$$

où $c$ est une constante positive absolue. Nous démontrons ici les deux propositions suivantes.

Proposition 2 (HR) On a $J_{\varepsilon} \ll \varepsilon$.

Proposition 3 (HR) Soit $\delta>0$. Pour $N \geqslant N_{0}(\delta)$ et $\varepsilon \geqslant 25(\log \log N)^{5 / 2+\delta}(\log N)^{-1 / 2}$, on a

$$
I_{N, \varepsilon} \ll N^{-\varepsilon / 2} .
$$

Le choix $\varepsilon=25(\log \log N)^{5 / 2+\delta}(\log N)^{-1 / 2}$ donne le théorème.

\section{$3 \quad$ Étude du quotient $\zeta(s) / \zeta(s+\varepsilon)$}

Dans ce paragraphe, nous étudions, sous l'hypothèse de Riemann, le comportement de la fonction $\zeta(s) / \zeta(s+\varepsilon)$ dans le demi-plan $\sigma \geqslant 1 / 2$, quand $\varepsilon$ tend vers 0 . Afin de préciser, sur certains points, l'exposé de Burnol dans [7, nous utilisons le produit de Hadamard de $\zeta(s)$ et majorons chaque facteur $\operatorname{de} \zeta(s) / \zeta(s+\varepsilon)$.

Nous supposons $0<\varepsilon \leqslant 1 / 2$. 
Proposition 4 (HR) On a les estimations suivantes.

$$
\begin{aligned}
& \text { (i) } \quad\left|\frac{\zeta(s)}{\zeta(s+\varepsilon)}\right|^{2} \ll|s|^{\varepsilon} \quad(\sigma=1 / 2) ; \\
& \text { (ii) } \quad\left|\frac{\zeta(s)}{\zeta(s+\varepsilon)}\right|^{2} \leqslant 1+O\left(\varepsilon|s|^{1 / 2}\right) \quad(\sigma=1 / 2) ; \\
& \text { (iii) } \quad \frac{\zeta(s) / \zeta(s+\varepsilon)}{s(1-s)} \ll \frac{|s|^{\varepsilon / 2}}{|s-1|^{2}} \quad(\sigma \geqslant 1 / 2, s \neq 1) .
\end{aligned}
$$

\section{Démonstration}

Si l'on pose

$$
\xi(s)=\frac{1}{2} s(s-1) \pi^{-s / 2} \Gamma(s / 2) \zeta(s),
$$

on a

$$
\xi(s)=\prod_{\rho}\left(1-\frac{s}{\rho}\right),
$$

où le produit porte sur les zéros non triviaux $\rho$ de la fonction $\zeta$, et doit être calculé par la formule $\prod_{\rho}=\lim _{T \rightarrow+\infty} \prod_{|\gamma| \leqslant T}$ (on pose $\rho=\beta+i \gamma$ ). Par conséquent

$$
\frac{\zeta(s)}{\zeta(s+\varepsilon)}=\pi^{-\varepsilon / 2} \frac{(s+\varepsilon)(s+\varepsilon-1)}{s(s-1)} \frac{\Gamma((s+\varepsilon) / 2)}{\Gamma(s / 2)} \prod_{\rho} \frac{s-\rho}{s+\varepsilon-\rho} .
$$

Examinons successivement les facteurs apparaissant dans (4). On a d'abord $\pi^{-\varepsilon / 2}<1$. Ensuite, on a

$$
\begin{aligned}
& \left|\frac{(s+\varepsilon)(s+\varepsilon-1)}{s(s-1)}\right| \ll\left|\frac{s}{s-1}\right| \quad(\sigma \geqslant 1 / 2, s \neq 1), \\
& \left|\frac{(s+\varepsilon)(s+\varepsilon-1)}{s(s-1)}\right| \leqslant \exp (O(\varepsilon /|s|)) \quad(\sigma=1 / 2) .
\end{aligned}
$$

Pour le quotient des fonctions $\Gamma$ apparaissant dans la formule (44), on dispose de l'inégalité suivante, qui résulte de la formule de Stirling complexe.

$$
\left|\frac{\Gamma((s+\varepsilon) / 2)}{\Gamma(s / 2)}\right| \leqslant|s / 2|^{\varepsilon / 2} \exp (O(\varepsilon /|s|)) \quad(\sigma \geqslant 1 / 2) .
$$

Pour majorer le produit infini apparaissant dans (4), on utilise l'inégalité

$$
\left|\frac{s-\rho}{s+\varepsilon-\rho}\right|<1, \quad \sigma \geqslant \beta, \quad \varepsilon>0,
$$

qui donne par conséquent (sous l'hypothèse de Riemann)

$$
\left|\prod_{\rho} \frac{s-\rho}{s+\varepsilon-\rho}\right|<1 \quad(\sigma \geqslant 1 / 2) .
$$


Notons ensuite les inégalités

$$
\exp (\varepsilon \log x / 2+O(\varepsilon / x)) \ll(x / 2)^{\varepsilon},
$$

et

$$
\exp (\varepsilon \log x / 2+O(\varepsilon / x)) \leqslant 1+O\left(\varepsilon x^{1 / 2}\right),
$$

valables pour $x \geqslant 1 / 2$.

L'estimation (i) résulte alors de (4), (6), (7), (8) et (9) ; l'estimation (ii) de (4), (6), (7), (8) et (10), et l'estimation (iii) de (4), (5), (7), (8) et (9).

\section{Majoration de $J_{\varepsilon}$}

On suppose, comme au $₫$, que $\varepsilon$ vérifie $0<\varepsilon \leqslant 1 / 2$. On pose

$$
K_{\varepsilon}=\frac{1}{2 \pi} \int_{\sigma=1 / 2}\left|\frac{\zeta(s)}{\zeta(s+\varepsilon)}\right|^{2} \frac{d \tau}{|s|^{2}} \quad \text { et } \quad L_{\varepsilon}=\frac{1}{2 \pi} \int_{\sigma=1 / 2} \frac{\zeta(s)}{\zeta(s+\varepsilon)} \frac{d \tau}{|s|^{2}},
$$

de sorte que

$$
J_{\varepsilon}=K_{\varepsilon}-2 L_{\varepsilon}+1
$$

Pour majorer $J_{\varepsilon}$, nous allons calculer exactement $L_{\varepsilon}$ à l'aide du théorème des résidus, et majorer $K_{\varepsilon}$ en utilisant les résultats du paragraphe précédent.

Proposition 5 (HR) On a

$$
\begin{aligned}
L_{\varepsilon} & =\frac{\gamma-1}{\zeta(1+\varepsilon)}-\frac{\zeta^{\prime}(1+\varepsilon)}{\zeta^{2}(1+\varepsilon)} \\
& =1-(\gamma+1) \varepsilon+O\left(\varepsilon^{2}\right) .
\end{aligned}
$$

\section{Démonstration}

On a

$$
\begin{aligned}
L_{\varepsilon} & =\frac{1}{2 \pi} \int_{\sigma=1 / 2} \frac{\zeta(s)}{\zeta(s+\varepsilon)} \frac{d \tau}{|s|^{2}} \\
& =\frac{1}{2 \pi i} \int_{\sigma=1 / 2} Q(s) d s,
\end{aligned}
$$

où

$$
Q(s)=\frac{\zeta(s) / \zeta(s+\varepsilon)}{s(1-s)} .
$$

Soit $\Pi$ le demi-plan $\sigma \geqslant \frac{1}{2}$, et $\Delta$ la droite $\sigma=\frac{1}{2}$. La fonction $Q$ est méromorphe dans $\Pi$, holomorphe $\operatorname{sur} \Delta$. Dans $\Pi$ elle a un unique pôle, double, en $s=1$ où son résidu vaut

$$
\frac{1-\gamma}{\zeta(1+\varepsilon)}+\frac{\zeta^{\prime}(1+\varepsilon)}{\zeta^{2}(1+\varepsilon)}
$$


D'après la proposition 4, (iii), on a $s Q(s) \rightarrow 0$ uniformément quand $|s| \rightarrow+\infty, s \in \Pi$, et

$$
\int_{\Delta}|Q(s)| \cdot|d s|<+\infty .
$$

Nous sommes donc en situation d'appliquer une proposition classique du calcul des résidus (cf. par exemple $[18] \S 6.22)$ pour en déduire

$$
\begin{aligned}
L_{\varepsilon} & =-\left.\operatorname{Res}\left(\frac{\zeta(s)}{\zeta(s+\varepsilon)} \cdot \frac{1}{s(1-s)}\right)\right|_{s=1} \\
& =\frac{\gamma-1}{\zeta(1+\varepsilon)}-\frac{\zeta^{\prime}(1+\varepsilon)}{\zeta^{2}(1+\varepsilon)} .
\end{aligned}
$$

Cette dernière quantité vaut

$$
1-(\gamma+1) \varepsilon+O\left(\varepsilon^{2}\right)
$$

puisque

$$
\frac{1}{\zeta(1+\varepsilon)}=\varepsilon-\gamma \varepsilon^{2}+O\left(\varepsilon^{3}\right) .
$$

Nous sommes maintenant en mesure de démontrer l'estimation $J_{\varepsilon} \ll \varepsilon$, objet de la proposition 2, En intégrant l'inégalité (ii) de la proposition 4 sur la droite $\sigma=1 / 2$ avec la mesure $d \tau /\left|s^{2}\right|$, on obtient

$$
K_{\varepsilon}-1 \ll \varepsilon .
$$

Le résultat découle alors de (11) et de la proposition [5.

En considérant la contribution à $J_{\varepsilon}$ d'un voisinage de l'ordonnée d'un zéro simple de $\zeta$ (par exemple $\gamma_{1}=14,1347 \ldots$ ), on peut montrer inconditionnellement que $J_{\varepsilon} \gg \varepsilon$. Il serait intéressant de préciser le comportement asymptotique de $J_{\varepsilon}$ quand $\varepsilon$ tend vers 0 .

\section{Quelques propriétés de la fonction $\zeta$ sous l'hypothèse de Rie- mann}

Afin d'établir la majoration de la proposition 3 nous allons étudier $M_{N}(s+\varepsilon)$. Pour cela, nous allons utiliser la méthode inventée par Maier et Montgomery dans l'article [12, dévolu à $M_{N}(0)=M(N)$. Ils y démontrent que

$$
M(N)=\sum_{n \leqslant N} \mu(n) \ll \sqrt{N} \exp \left((\log N)^{39 / 61}\right)
$$

sous l'hypothèse de Riemann. Leur approche a été ensuite perfectionnée par Soundararajan (cf. [14]), qui a obtenu l'estimation

$$
M(N) \ll \sqrt{N} \exp \left((\log N)^{1 / 2}(\log \log N)^{14}\right),
$$

toujours sous l'hypothèse de Riemann. La méthode de Soundararajan donne en fait

$$
M(N) \ll_{\delta} \sqrt{N} \exp \left((\log N)^{1 / 2}(\log \log N)^{5 / 2+\delta}\right),
$$

pour tout $\delta$ tel que $0<\delta \leqslant 1 / 2$. Nous allons maintenant rappeler les éléments de la méthode de Soundararajan qui seront utilisés dans notre argumentation, avec les quelques modifications qui permettent d'obtenir l'exposant $5 / 2+\delta$. On trouvera les démonstrations dans l'article [14] (cf. aussi [5] pour un exposé détaillé des modifications). 


\subsection{Ordonnées $V$-typiques}

L'évaluation de $M_{N}(s+\varepsilon)$ grâce à la formule de Perron fera appel à un contour sur lequel les grandes valeurs de $|\zeta(z)|^{-1}$ seront aussi rares que possible. Pour quantifier cette rareté, Soundararajan a introduit la notion suivante.

Soit $T$ assez grand đ et $V$ tel que $(\log \log T)^{2} \leqslant V \leqslant \log T / \log \log T$. Un nombre réel $t$ est appelé une ordonnée $V$-typique de taille $T$ si

- $T \leqslant t \leqslant 2 T$;

(i) pour tout $\sigma \geqslant 1 / 2$, on a

$$
\left|\sum_{n \leqslant x} \frac{\Lambda(n)}{n^{\sigma+i t} \log n} \frac{\log (x / n)}{\log x}\right| \leqslant 2 V, \quad \text { où } x=T^{1 / V}
$$

(ii) tout sous-intervalle de $[t-1, t+1]$ de longueur $2 \pi \delta V / \log T$ contient au plus $(1+\delta) V$ ordonnées de zéros de $\zeta$;

(iii) tout sous-intervalle de $[t-1, t+1]$ de longueur $2 \pi V /((\log V) \log T)$ contient au plus $V$ ordonnées de zéros de $\zeta$.

Si $t \in[T, 2 T]$ ne vérifie pas l'une des assertions (i), (ii), (iii), on dira que $t$ est une ordonnée $V$-atypique de taille $T$.

L'apport de cette définition à l'estimation de $M_{N}(s+\varepsilon)$ via la formule de Perron (\$ contenu dans l'énoncé suivant (proposition 9 de [5]).

Proposition 6 (HR) Soitt assez grand, et $x \geqslant t$. Soit $V^{\prime}$ tel que $(\log \log t)^{2} \leqslant V^{\prime} \leqslant(\log t / 2) /(\log \log t / 2)$. On suppose que $t$ est une ordonnée $V^{\prime}$-typique (de taille $T^{\prime}$ ). Soit $V \geqslant V^{\prime}$.

Alors

$$
\left|x^{z} \zeta(z)^{-1}\right| \leqslant \sqrt{x} \exp (V \log (\log x / \log t)+(2+3 \delta) V \log \log V) \quad\left(V^{\prime} \leqslant(\Re z-1 / 2) \log x \leqslant V, \quad|\Im z|=t\right) .
$$

\subsection{Majoration de l'écart entre le nombre de zéros de la fonction $\zeta$ et sa moyenne, dans un intervalle de la droite critique}

La proposition suivante (cf. [5], proposition 15) donne une majoration de l'écart entre le nombre d'ordonnées de zéros de $\zeta$ dans l'intervalle $] t-h, t+h]$ et sa valeur moyenne $(h / \pi) \log (t / 2 \pi)$. Cet encadrement est exprimé au moyen d'un paramètre $\Delta$, et met notamment en jeu un polynôme de Dirichlet de longueur $\exp 2 \pi \Delta$.

Proposition 7 (HR) Soit $\Delta \geqslant 2$ et $h>0$. Il existe des nombres réels a $(p)=a(p, \Delta, h)$ (p premier, $\left.p \leqslant e^{2 \pi \Delta}\right)$ vérifiant

- $|a(p)| \leqslant 4$ pour $p \leqslant e^{2 \pi \Delta}$;

- pour tout $t$ tel que $t \geqslant \max \left(4, h^{2}\right)$, on a

$$
N(t+h)-N(t-h)-2 h \frac{\log t / 2 \pi}{2 \pi} \leqslant \frac{\log t}{2 \pi \Delta}+\sum_{p \leqslant e^{2 \pi \Delta}} \frac{a(p) \cos (t \log p)}{p^{\frac{1}{2}}}+O(\log \Delta) .
$$

\footnotetext{
${ }^{\dagger}$ Ici et dans la suite, cela signifie que $T \geqslant T_{0}(\delta)$, quantité effectivement calculable, et dépendant au plus de $\delta$.
} 
Lorsqu'on majore trivialement le polynôme de Dirichlet qui intervient dans cette proposition, on obtient le résultat suivant, dû à Goldston et Gonek (cf. [10]). Notre énoncé est légèrement plus précis que celui de [10.

Proposition 8 Soit $t$ assez grand et $0<h \leqslant \sqrt{t}$. On a

$$
N(t+h)-N(t-h)-(h / \pi) \log (t / 2 \pi) \leqslant(\log t) / 2 \log \log t+(1 / 2+o(1)) \log t \log \log \log t /(\log \log t)^{2} .
$$

\section{Démonstration}

On a

$$
\begin{aligned}
\left|\sum_{p \leqslant e^{2 \pi \Delta}} \frac{a(p) \cos t \log p}{p^{\frac{1}{2}}}\right| & \ll \sum_{p \leqslant e^{2 \pi \Delta}} \frac{1}{\sqrt{p}} \\
& \ll \frac{e^{\pi \Delta}}{\Delta} .
\end{aligned}
$$

On choisit $\Delta=\frac{1}{\pi} \log (\log t / \log \log t)$ et on vérifie alors que

$$
\frac{\log t}{2 \pi \Delta}+O\left(e^{\pi \Delta} / \Delta\right)+O(\log \Delta)=(\log t) / 2 \log \log t+(1 / 2+o(1)) \log t \log \log \log t /(\log \log t)^{2} .
$$

La proposition suivante est une variante un peu plus précise de la première assertion de la Proposition 4 de [14.

Proposition 9 Soit $T$ assez grand, et $V$ tel que

$$
\frac{1}{2}+\left(\frac{1}{2}+\delta\right) \log \log \log T / \log \log T \leqslant V \log \log T / \log T \leqslant 1 .
$$

Alors toute ordonnée $t \in[T, 2 T]$ est $V$-typique.

\section{Démonstration}

Il faut vérifier les critères (i), (ii), (iii) de la définition d'une ordonnée $V$-typique.

Pour (i), on a pour $\sigma \geqslant 1 / 2, t \in \mathbb{R}$, et $x=T^{1 / V}$,

$$
\begin{aligned}
\left|\sum_{n \leqslant x} \frac{\Lambda(n)}{n^{\sigma+i t} \log n} \frac{\log (x / n)}{\log x}\right| & \leqslant \sum_{n \leqslant x} \frac{\Lambda(n)}{\sqrt{n} \log n} \frac{\log (x / n)}{\log x} \\
& \ll \frac{\sqrt{x}}{(\log x)^{2}} \\
& \ll \frac{\log T}{(\log \log T)^{2}} \quad\left(\operatorname{car} x=T^{1 / V} \leqslant(\log T)^{2}\right) \\
& =o(V) .
\end{aligned}
$$

Pour (ii) on a, avec $t^{\prime} \in[t-1, t+1]$ et $h=\pi \delta V / \log T$ :

$$
\begin{aligned}
N\left(t^{\prime}+h\right)-N\left(t^{\prime}-h\right) \leqslant & (h / \pi) \log \left(t^{\prime} / 2 \pi\right)+\frac{1}{2} \log t^{\prime} / \log \log t^{\prime}+(1 / 2+o(1)) \log t^{\prime} \log \log \log t^{\prime} /\left(\log \log t^{\prime}\right)^{2} \\
& (\text { proposition } \underline{8}) \\
\leqslant & (h / \pi) \log T+\frac{1}{2} \log T / \log \log T+(1 / 2+\delta) \log T \log \log \log T /(\log \log T)^{2} \\
\leqslant & (1+\delta) V .
\end{aligned}
$$


Pour (iii) on a, avec $t^{\prime} \in[t-1, t+1]$ et $h=\pi V /((\log V) \log T)$ :

$$
\begin{aligned}
N\left(t^{\prime}+h\right)-N\left(t^{\prime}-h\right) & \leqslant(h / \pi) \log \left(t^{\prime} / 2 \pi\right)+\frac{1}{2} \log t^{\prime} / \log \log t^{\prime}+(1 / 2+o(1)) \log t^{\prime} \log \log \log t^{\prime} /\left(\log \log t^{\prime}\right)^{2} \\
& \leqslant \frac{V}{\log V}+\frac{1}{2} \log T / \log \log T+(1 / 2+o(1)) \log T \log \log \log T /(\log \log T)^{2} \\
& \leqslant \frac{1}{2} \log T / \log \log T+(1 / 2+\delta) \log T \log \log \log T /(\log \log T)^{2} \\
& \leqslant V
\end{aligned}
$$

\section{Approximation de l'inverse de la fonction $\zeta$ par ses sommes partielles}

Le but de ce paragraphe est la démonstration de la proposition suivante.

Proposition 10 Soit $N$ assez grand et $\varepsilon \geqslant 25(\log \log N)^{5 / 2+6 \delta}(\log N)^{-1 / 2}$. Alors, pour $|\tau| \leqslant N^{3 / 4}$, on a

$$
\zeta(s+\varepsilon)^{-1}-M_{N}(s+\varepsilon) \ll N^{-\varepsilon / 4}(1+|\tau|)^{1 / 2-\beta(\tau)},
$$

où $\beta(\tau)=\frac{\log \log \log (16+|\tau|)}{2 \log \log (16+|\tau|)}$.

Elle résultera de diverses estimations, valables uniformément quand $\tau$ et $\varepsilon$ appartiennent à certains intervalles définis en termes de $N$, longueur du polynôme de Dirichlet $M_{N}$, approximant la fonction $\zeta^{-1}$.

Pour plus de clarté dans l'exposé, nous développons séparément les analyses relatives aux deux paramètres $\tau$ et $\varepsilon$. Nous commençons par l'étude de

$$
M_{N}(i \tau)=\sum_{n \leqslant N} \mu(n) n^{-i \tau}
$$

pour $\tau \in \mathbb{R}$.

\subsection{Estimation de $M_{N}(i \tau)$ pour les petites valeurs de $|\tau|$}

Commençons par le résultat obtenu par sommation partielle à partir de la majoration de Soundararajan (cf. [14] et [5])

$$
M(x)=\sum_{n \leqslant x} \mu(n) \ll \sqrt{x} \exp C(\log x), \quad x \geqslant 3,
$$

où $C(u)=u^{1 / 2}(\log u)^{5 / 2+\delta}$. Observons que $C^{\prime}(u)=O(1), u \geqslant 1$.

Proposition 11 On a uniformément

$$
M_{N}(i \tau) \ll(1+|\tau|) \sqrt{N} \exp C(\log N), \quad N \geqslant 3, \quad \tau \in \mathbb{R} .
$$

La démonstration (standard) est laissée au lecteur. Pour aller plus loin, nous allons appliquer la formule de Perron et suivre la démarche de Soundararajan dans [14]. 


\subsection{Estimation de $M_{N}(i \tau)$ pour les grandes valeurs de $|\tau|$}

Nous utiliserons la majoration simple suivante.

Proposition 12 Pour $0<\delta \leqslant 1 / 12, N$ assez grand et

$$
\exp \left(3(\log N)^{1 / 2}(\log \log N)^{5 / 2+6 \delta}\right) \leqslant|\tau| \leqslant N^{3 / 4}
$$

on $a$

$$
M_{N}(i \tau) \ll N^{1 / 2}|\tau|^{1 / 2-\kappa(\tau)},
$$

ò̀ $\kappa(\tau)=\frac{1}{2} \log \log \log |\tau| / \log \log |\tau|$.

Démonstration Dans toute la démonstration, $N$ sera supposé assez grand.

\section{Première étape : formule de Perron}

La première étape de la démonstration consiste à appliquer la formule de Perron à la hauteur $N_{1}=$ $2^{\lfloor\log N / \log 2\rfloor}$ (le choix d'une puissance de 2 simplifie l'exposé de [5]), ce qui pour $\tau \in \mathbb{R}$ donne

$$
\begin{aligned}
M_{N}(i \tau) & =\frac{1}{2 \pi i} \int_{1+1 / \log N-i N_{1}}^{1+1 / \log N+i N 1} \zeta(z+i \tau)^{-1} \frac{N^{z}}{z} d z+O\left(N \log N_{1} / N_{1}\right) \\
& =\frac{1}{2 \pi i} \int_{1+1 / \log N-i\left(N_{1}-\tau\right)}^{1+1 / \log N+i\left(N_{1}+\tau\right)} \zeta(z)^{-1} \frac{N^{z-i \tau}}{z-i \tau} d z+O(\log N)
\end{aligned}
$$

Supposons maintenant que $|\tau| \leqslant N / 5$ et remplaçons l'intégrale par $N^{-i \tau} B_{N}$, où

$$
B_{N}=B_{N}(i \tau)=\frac{1}{2 \pi i} \int_{1+1 / \log N-i N_{1}}^{1+1 / \log N+i N_{1}} \zeta(z)^{-1} \frac{N^{z}}{z-i \tau} d z .
$$

L'erreur commise est alors majorée par

$$
\frac{1}{2 \pi} \int_{N_{1}-|\tau| \leqslant|\Im z| \leqslant N_{1}+|\tau|}\left|\zeta(z)^{-1}\right|\left|\frac{N^{z} d z}{z-i \tau}\right| \quad(\Re z=1+1 / \log N) .
$$

Or $\left|\zeta(z)^{-1}\right| \ll \log N$ si $\Re z=1+1 / \log N$ et $|z-i \tau| \gg N$ si $N_{1}-|\tau| \leqslant|\Im z| \leqslant N_{1}+|\tau|$, donc l'erreur est $O(|\tau| \log N)$.

Pour $N \geqslant 3$ et $|\tau| \leqslant N / 5$ on a donc montré

$$
M_{N}(i \tau)=N^{-i \tau} B_{N}+O((1+|\tau|) \log N) .
$$

\section{Deuxième étape : déformation du chemin d'intégration}

Pour majorer $\left|B_{N}\right|$, nous allons remplacer le segment d'intégration $\left[1+1 / \log N-i N_{1}, 1+1 / \log N+i N_{1}\right]$ par une variante $\mathcal{S}_{N}$ du chemin défini par Soundararajan dans [14, chemin sur lequel les grandes valeurs de l'intégrande sont rares. Nous commençons par une description de $\mathcal{S}_{N}$. Nous posons

$$
\kappa=\left\lfloor(\log N)^{1 / 2}(\log \log N)^{5 / 2}\right\rfloor, \quad K=\lfloor\log N / \log 2\rfloor .
$$


Nous posons également $T_{k}=2^{k}$ pour $\kappa \leqslant k \leqslant K$, et $N_{0}=T_{\kappa}$ (on a $N_{1}=T_{K}$ ).

Le chemin $\mathcal{S}_{N}$ est symétrique par rapport à l'axe réel, et constitué de segments verticaux et horizontaux. Nous décrivons seulement la partie de $\mathcal{S}_{N}$ située dans le demi-plan $\Im z \geqslant 0$.

- Il y a d'abord un segment vertical $\left[1 / 2+1 / \log N, 1 / 2+1 / \log N+i N_{0}\right]$.

- Pour chaque $k$ tel que $\kappa \leqslant k<K$, on considère les entiers $n$ de l'intervalle $\left[T_{k}, 2 T_{k}[\right.$. On définit alors $V_{n}$ comme le plus petit entier de l'intervalle [ $\left.\left(\log \log T_{k}\right)^{2}, \log T_{k} / \log \log T_{k}\right]$ tel que tous les points de $[n, n+1]$ soient $V_{n}$-typiques de taille $T_{k}$. L'existence de $V_{n}$ est garantie par la proposition 9] On a même

$$
V_{n} \leqslant \frac{1}{2} \log n / \log \log n+(1 / 2+\delta) \log n(\log \log \log n) /(\log \log n)^{2}+1 .
$$

On inclut alors dans $\mathcal{S}_{N}$ le segment vertical $\left[1 / 2+V_{n} / \log N+i n, 1 / 2+V_{n} / \log N+i(n+1)\right]$

Il y a enfin des segments horizontaux reliant tous ces segments verticaux :

- le segment $\left[1 / 2+1 / \log N+i N_{0}, 1 / 2+V_{N_{0}} / \log N+i N_{0}\right]$;

- les segments $\left[1 / 2+V_{n} / \log N+i(n+1), 1 / 2+V_{n+1} / \log N+i(n+1)\right], N_{0} \leqslant n \leqslant T_{K}-2$;

- le segment $\left[1 / 2+V_{N_{1}-1} / \log N+i N_{1}, 1+1 / \log N+i N_{1}\right]$.

D'après le théorème de Cauchy, on a

$$
B_{N}=\frac{1}{2 i \pi} \int_{\mathcal{S}_{N}} \zeta(z)^{-1} \frac{N^{z}}{z-i \tau} d z .
$$

\section{Troisième étape : évaluation de $B_{N}$}

Lorsque $|z-i \tau|$ n'est pas trop petit devant $|z|$, nous pouvons utiliser les estimations de [14] et [5]. Nous définissons donc $\mathcal{S}_{N, \tau}$ comme la partie de $\mathcal{S}_{N}$ où $|(\Im z-\tau) / \tau| \leqslant 1 / 4(\tau \neq 0)$.

Si $z \in \mathcal{S}_{N} \backslash \mathcal{S}_{N, \tau}$, on a $|z-i \tau| \gg|z|$. Par conséquent (cf. [14] et [5]), pour $N \geqslant 3$ et $\tau \in \mathbb{R}$, on a

$$
\begin{aligned}
\left|B_{N}-\frac{1}{2 i \pi} \int_{\mathcal{S}_{N, \tau}} \zeta(z)^{-1} \frac{N^{z}}{z-i \tau} d z\right| & \ll \int_{\mathcal{S}_{N}}\left|\frac{\zeta(z)^{-1} N^{z} d z}{z}\right| \\
& \ll \sqrt{N} \exp \left((\log N)^{1 / 2}(\log \log N)^{5 / 2+6 \delta}\right) .
\end{aligned}
$$

Il nous reste à majorer la contribution de $\mathcal{S}_{N, \tau}$.

Supposons $\sqrt{2} N_{0} \leqslant|\tau| \leqslant \frac{1}{\sqrt{2}} N_{1}$. Par symétrie, on peut également supposer $\tau>0$. On a

$$
\left|\frac{1}{2 i \pi} \int_{\mathcal{S}_{N, \tau}} \zeta(z)^{-1} \frac{N^{z}}{z-i \tau} d z\right| \leqslant \sup _{z \in \mathcal{S}_{N, \tau}}\left|\zeta(z)^{-1} N^{z}\right|\left(\frac{1}{2 \pi} \int_{\mathcal{S}_{N, \tau}}\left|\frac{d z}{z-i \tau}\right|\right) .
$$

Observons que si $z \in \mathcal{S}_{N}$ et $\Im z \geqslant N_{0}$, alors $z$ se trouve sur un des segments horizontaux et verticaux décrits ci-dessus. Sur les deux segments (horizontal et vertical) de $\mathcal{S}_{N, \tau}$ situés dans la bande $n<\Im z \leqslant n+1$, on a $|z-i \tau|^{-1} \ll(1+|n-\tau|)^{-1}$, donc l'intégrale est en $O(\log \tau)$.

Pour majorer $\left|\zeta(z)^{-1} N^{z}\right|$, nous utilisons la proposition [6. En posant $n=\lceil\Im z\rceil-1$, on peut écrire

$$
V^{\prime} \leqslant(\Re z-1 / 2) \log N \leqslant V
$$


avec $\left(V, V^{\prime}\right)=\left(V_{n}, V_{n}\right)$ dans le cas vertical et $\left(V_{n+1}, V_{n}\right)$ ou $\left(V_{n}, V_{n+1}\right)$ dans le cas horizontal $(\Im z=n+1)$, et $\Im z V^{\prime}$-typique (de taille correspondante). On peut donc bien appliquer la proposition 6 pour obtenir

$$
\left|\zeta(z)^{-1} N^{z}\right| \leqslant \sqrt{N} \exp (V \log (\log N / \log \Im z)+(2+3 \delta) V \log \log V) .
$$

Maintenant, si $z \in \mathcal{S}_{N, \tau}$, on a

$$
\tau \sqrt{2} \geqslant \Im z \geqslant \tau / \sqrt{2} \geqslant N_{0}
$$

donc

$$
\log N / \log \Im z \leqslant \log \Im z \leqslant \log \tau \sqrt{2}
$$

D'autre part,

$$
\begin{aligned}
V & \leqslant \frac{1}{2} \log (n+1) / \log \log (n+1)+(1 / 2+\delta) \log (n+1) \log \log \log (n+1) /(\log \log (n+1))^{2}+1 \\
& \leqslant \frac{1}{2} \log \tau / \log \log \tau+(1 / 2+2 \delta) \log \tau \log \log \log \tau /(\log \log \tau)^{2} .
\end{aligned}
$$

Par conséquent,

$$
\begin{aligned}
V \log (\log N / \log \Im z)+(2+3 \delta) V \log \log V \leqslant & \frac{1}{2}(\log \tau / \log \log \tau) \log (\log N / \log \tau) \\
& +(3 / 2+5 \delta) \log \tau \log \log \log \tau / \log \log \tau .
\end{aligned}
$$

On a donc montré que

$\sup _{z \in \mathcal{S}_{N, \tau}}\left|\zeta(z)^{-1} N^{z}\right| \leqslant \sqrt{N} \exp \left(\frac{1}{2}(\log \tau / \log \log \tau) \log (\log N / \log \tau)+(3 / 2+5 \delta) \log \tau \log \log \log \tau / \log \log \tau\right)$.

Ainsi, pour $\sqrt{2} N_{0} \leqslant|\tau| \leqslant \frac{1}{\sqrt{2}} N_{1}$, on a

$$
\begin{aligned}
\frac{1}{2 i \pi} \int_{\mathcal{S}_{N, \tau}} \zeta(z)^{-1} \frac{N^{z}}{z-i \tau} d z \leqslant & \sqrt{N} \exp ((\log |\tau| / 2 \log \log |\tau|) \log (\log N / \log |\tau|) \\
& +(3 / 2+6 \delta) \log |\tau| \log \log \log |\tau| / \log \log |\tau|)
\end{aligned}
$$

ce qui donne finalement, en utilisant (13)

$$
\begin{aligned}
B_{N} \leqslant & \sqrt{N} \exp \left(\frac{1}{2}(\log |\tau| / \log \log |\tau|) \log (\log N / \log |\tau|)+(3 / 2+6 \delta) \log |\tau| \log \log \log |\tau| / \log \log |\tau|\right) \\
& +O\left(\sqrt{N} \exp \left((\log N)^{1 / 2}(\log \log N)^{5 / 2+6 \delta}\right)\right)
\end{aligned}
$$

\section{Conclusion : estimation de $M_{N}(i \tau)$}

D'après (12) et (14), on a

$$
M_{N}(i \tau)=N^{-i \tau} B_{N}+O(|\tau| \log N) \quad(1 \leqslant|\tau| \leqslant N / 5)
$$


et

$$
\begin{aligned}
B_{N} \leqslant & \sqrt{N} \exp \left(\frac{1}{2}(\log |\tau| / \log \log |\tau|) \log (\log N / \log |\tau|)+(3 / 2+6 \delta) \log |\tau| \log \log \log |\tau| / \log \log |\tau|\right) \\
& +O\left(\sqrt{N} \exp \left((\log N)^{1 / 2}(\log \log N)^{5 / 2+6 \delta}\right)\right) .
\end{aligned}
$$

On observe que sous les hypothèses de la proposition, on a :

$$
|\tau| \log N \leqslant N^{1 / 2}|\tau|^{2 / 5}
$$

et

$$
N^{1 / 2} \exp \left((\log N)^{1 / 2}(\log \log N)^{5 / 2+6 \delta}\right) \leqslant N^{1 / 2}|\tau|^{1 / 3}
$$

On a également

$$
\begin{aligned}
\frac{\log |\tau|}{(\log \log |\tau|)^{5 / 2}} & \geqslant \frac{3(\log N)^{1 / 2}(\log \log N)^{5 / 2}}{\left(\log \left(3(\log N)^{1 / 2}(\log \log N)^{5 / 2}\right)\right)^{5 / 2}} \\
& \geqslant \sqrt{\log N}
\end{aligned}
$$

Par conséquent,

$$
\frac{\log N}{\log |\tau|} \leqslant \frac{\log |\tau|}{(\log \log |\tau|)^{5}}
$$

ce qui implique

$$
\frac{1}{2} \frac{\log |\tau|}{\log \log |\tau|} \cdot \log \left(\frac{\log N}{\log |\tau|}\right)+(3 / 2+6 \delta) \log |\tau| \frac{\log \log \log |\tau|}{\log \log |\tau|} \leqslant \frac{1}{2} \log |\tau|+(-1+6 \delta) \log |\tau| \frac{\log \log \log |\tau|}{\log \log |\tau|}
$$

et permet de conclure.

\subsection{Estimations de $\zeta(s+\varepsilon)^{-1}-M_{N}(s+\varepsilon)$}

Démontrons à présent la proposition 10 et revenons à l'estimation de la différence

$$
\zeta(s+\varepsilon)^{-1}-M_{N}(s+\varepsilon),
$$

que nous exprimons d'abord à l'aide d'une intégrale :

$$
\zeta(s+\varepsilon)^{-1}-M_{N}(s+\varepsilon)=-M_{N}(i \tau) N^{-1 / 2-\varepsilon}+(1 / 2+\varepsilon) \int_{N}^{\infty} t^{-3 / 2-\varepsilon} M_{t}(i \tau) d t \quad(N \geqslant 1, \varepsilon>0, \tau \in \mathbb{R})
$$

On suppose $N$ assez grand, $\varepsilon \geqslant 2(\log \log N)^{5 / 2+\delta}(\log N)^{-1 / 2}$, et $\tau \in \mathbb{R}$. 
Petites valeurs de $|\tau|$

On a d'abord, d'après la proposition 11

$$
M_{N}(i \tau) N^{-1 / 2-\varepsilon} \ll(1+|\tau|) N^{-\varepsilon} \exp \left((\log N)^{1 / 2}(\log \log N)^{5 / 2+\delta}\right) .
$$

D'autre part, pour $t \geqslant N$, on a

$$
\frac{\varepsilon}{2} \log t \geqslant(\log t)^{1 / 2}(\log \log t)^{5 / 2+\delta} .
$$

En particulier,

$$
M_{N}(i \tau) N^{-1 / 2-\varepsilon} \ll(1+|\tau|) N^{-\varepsilon / 2}
$$

Et aussi,

$$
\begin{aligned}
\int_{N}^{\infty} t^{-3 / 2-\varepsilon} M_{t}(i \tau) d t & \ll(1+|\tau|) \int_{N}^{\infty} t^{-1-\varepsilon} \exp \left((\log t)^{1 / 2}(\log \log t)^{5 / 2+\delta}\right) d t \\
& \leqslant(1+|\tau|) \int_{N}^{\infty} t^{-1-\varepsilon / 2} d t \\
& \ll \varepsilon^{-1}(1+|\tau|) N^{-\varepsilon / 2}
\end{aligned}
$$

Or

$$
\begin{aligned}
\varepsilon^{-1} & \leqslant(\log \log N)^{-5 / 2}(\log N)^{1 / 2} \\
& \leqslant \exp \left(\frac{1}{3}(\log N)^{1 / 2}(\log \log N)^{5 / 2+\delta}\right) \\
& \leqslant N^{\varepsilon / 6}
\end{aligned}
$$

donc $\varepsilon^{-1} N^{-\varepsilon / 2} \ll N^{-\varepsilon / 3}$, ce qui donne sous nos hypothèses, la majoration

$$
\zeta(s+\varepsilon)^{-1}-M_{N}(s+\varepsilon) \ll(1+|\tau|) N^{-\varepsilon / 3} .
$$

Dans le cas $\exp \left(3(\log N)^{1 / 2}(\log \log N)^{5 / 2+6 \delta}\right) \geqslant|\tau|$, pour obtenir le résultat de la proposition 10, il nous suffit donc de démontrer que

$$
(1+|\tau|) N^{-\varepsilon / 3} \ll(1+|\tau|)^{1 / 3} N^{-\varepsilon / 4},
$$

c'est-à-dire

$$
\frac{\varepsilon}{12} \log N \geqslant \frac{2}{3} \log (1+|\tau|) .
$$

Or on a bien dans ce cas

$$
\begin{aligned}
\frac{2}{3} \log (1+|\tau|) & \leqslant \frac{2}{3}\left(3(\log N)^{1 / 2}(\log \log N)^{5 / 2+6 \delta}+O(1)\right) \\
& \leqslant \frac{25}{12}(\log N)^{1 / 2}(\log \log N)^{5 / 2+6 \delta} \\
& \leqslant \frac{\varepsilon}{12} \log N .
\end{aligned}
$$




\section{Grandes valeurs de $|\tau|$}

Si $\exp \left(3(\log N)^{1 / 2}(\log \log N)^{5 / 2+6 \delta}\right) \leqslant|\tau| \leqslant N^{3 / 4}$, on a d'abord, d'après la proposition 12

$$
M_{N}(i \tau) N^{-1 / 2-\varepsilon} \ll N^{-\varepsilon}|\tau|^{1 / 2-\kappa(\tau)} .
$$

Étudions maintenant l'intégrale

$$
\int_{N}^{\infty} t^{-3 / 2-\varepsilon} M_{t}(i \tau) d t
$$

Pour commencer, observons que $|\tau| \leqslant N^{3 / 4} \leqslant t^{3 / 4}$ si $t \geqslant N$.

D'autre part, définissons $\theta=\theta(\tau)$ par la relation

$$
|\tau|=\exp \left(3(\log \theta)^{1 / 2}(\log \log \theta)^{5 / 2+6 \delta}\right) .
$$

On a $\theta \geqslant N$ si $|\tau| \geqslant \exp \left(3(\log N)^{1 / 2}(\log \log N)^{5 / 2+6 \delta}\right)$, et

$$
\int_{N}^{\infty} t^{-3 / 2-\varepsilon} M_{t}(i \tau) d t=\int_{N}^{\theta} t^{-3 / 2-\varepsilon} M_{t}(i \tau) d t+\int_{\theta}^{\infty} t^{-3 / 2-\varepsilon} M_{t}(i \tau) d t .
$$

Pour la première intégrale, nous pouvons utiliser la proposition $12 \operatorname{car} t \leqslant \theta \Rightarrow|\tau| \geqslant \exp \left(3(\log t)^{1 / 2}(\log \log t)^{5 / 2+6 \delta}\right)$. Ainsi,

$$
\begin{aligned}
\int_{N}^{\theta} t^{-3 / 2-\varepsilon} M_{t}(i \tau) d t & \ll|\tau|^{1 / 2-\kappa(\tau)} \int_{N}^{\theta} t^{-1-\varepsilon} d t \\
& \leqslant|\tau|^{1 / 2-\kappa(\tau)} \varepsilon^{-1} N^{-\varepsilon} \\
& \leqslant|\tau|^{1 / 2-\kappa(\tau)} N^{-5 \varepsilon / 6}
\end{aligned}
$$

comme dans le cas précédent.

Pour la seconde intégrale, nous pouvons utiliser la proposition 11. On a

$$
\int_{\theta}^{\infty} t^{-3 / 2-\varepsilon} M_{t}(i \tau) d t \ll|\tau| \int_{\theta}^{\infty} t^{-1-\varepsilon} \exp \left((\log t)^{1 / 2}(\log \log t)^{5 / 2+6 \delta}\right) d t .
$$

Maintenant, pour $t \geqslant \theta(\tau)(\geqslant N)$, on a

$$
\frac{\varepsilon}{2} \log t \geqslant 4(\log t)^{1 / 2}(\log \log t)^{5 / 2+6 \delta} .
$$

Ainsi,

$$
\begin{aligned}
\int_{\theta}^{\infty} t^{-3 / 2-\varepsilon} M_{t}(i \tau) d t & \ll|\tau| \int_{\theta}^{\infty} t^{-1-\varepsilon / 2} \exp \left(-3(\log t)^{1 / 2}(\log \log t)^{5 / 2+6 \delta}\right) d t \\
& \leqslant|\tau| \exp \left(-3(\log \theta)^{1 / 2}(\log \log \theta)^{5 / 2+6 \delta}\right) \int_{\theta}^{\infty} t^{-1-\varepsilon / 2} d t \\
& =(2 / \varepsilon) \theta^{-\varepsilon / 2} \\
& \leqslant(2 / \varepsilon) N^{-\varepsilon / 2} \\
& \ll N^{-\varepsilon / 3}
\end{aligned}
$$


ce qui entraîne

$$
\zeta(s+\varepsilon)^{-1}-M_{N}(s+\varepsilon) \ll N^{-\varepsilon / 3}|\tau|^{1 / 2-\kappa(\tau)}
$$

Notons à présent que pour $|\tau|$ grand, on a $\beta(\tau)-\kappa(\tau) \ll 1 / \log |\tau|$. Cela permet de conclure la démonstration de la proposition 10.

\section{$7 \quad$ Majoration de $I_{N, \varepsilon}$}

Dans tout ce paragraphe, on pose $\sigma=\frac{1}{2}$, c'est-à-dire $s=\frac{1}{2}+i \tau$.

Proposition 13 (HR) Pour $N \geqslant 1,0<\varepsilon \leqslant 1 / 2$, on a

$$
\int_{|\tau| \geqslant N^{3 / 4}}|\zeta(s)|^{2}\left|\zeta(s+\varepsilon)-M_{N}(s+\varepsilon)\right|^{2} \frac{d \tau}{|s|^{2}} \ll N^{-1 / 9} .
$$

\section{Démonstration}

Il suffit de démontrer que, pour $T \geqslant 1$,

$$
I_{N}(T, \varepsilon)=\int_{T \leqslant|\tau| \leqslant 2 T}|\zeta(s)|^{2}\left|\zeta(s+\varepsilon)^{-1}-M_{N}(s+\varepsilon)\right|^{2} \frac{d \tau}{|s|^{2}} \ll T^{-3 / 2}(T+N) \log N,
$$

car (16) résultera de la sommation de (17) pour les valeurs $T=2^{k} N^{3 / 4}, k \in \mathbb{N}$.

On a

$$
I_{N}(T, \varepsilon) \ll T^{-2} \int_{T \leqslant \tau \leqslant 2 T}|\zeta(s) / \zeta(s+\varepsilon)|^{2} d \tau+4 T^{-2} \int_{T \leqslant \tau \leqslant 2 T}|\zeta(s)|^{2}\left|M_{N}(s+\varepsilon)\right|^{2} d \tau .
$$

D'une part,

$$
\int_{T \leqslant \tau \leqslant 2 T}|\zeta(s) / \zeta(s+\varepsilon)|^{2} d \tau \ll T^{3 / 2},
$$

d'après le point (i) de la proposition 4

D'autre part,

$$
\int_{T \leqslant \tau \leqslant 2 T}|\zeta(s)|^{2}\left|M_{N}(s+\varepsilon)\right|^{2} d \tau \leqslant T^{1 / 2} \int_{T \leqslant \tau \leqslant 2 T}\left|\sum_{n \leqslant N} \mu(n) n^{-1 / 2-\varepsilon} n^{-i \tau}\right|^{2} d \tau,
$$

d'après l'inégalité $|\zeta(s)| \ll \tau^{1 / 4}$ (cf. [16], (5.1.8) p.96).

La dernière intégrale vaut

$$
(T+O(N)) \sum_{n \leqslant N} \mu^{2}(n) n^{-1-2 \varepsilon} \leqslant(T+N) \log N
$$

d'après une inégalité de Montgomery et Vaughan (cf. [13], (5) p.128), et car $\sum_{n \leqslant N} n^{-1-2 \varepsilon} \ll \log N$. Par conséquent,

$$
I_{N, \varepsilon} \ll T^{-3 / 2}(T+N) \log N .
$$


Proposition 14 (HR) Soit $N$ assez grand et $\varepsilon \geqslant 25(\log \log N)^{5 / 2+6 \delta}(\log N)^{-1 / 2}$. Alors,

$$
\int_{|\tau| \leqslant N^{3 / 4}}|\zeta(s)|^{2}\left|\zeta(s+\varepsilon)^{-1}-M_{N}(s+\varepsilon)\right|^{2} \frac{d \tau}{|s|^{2}} \ll N^{-\varepsilon / 2} .
$$

\section{Démonstration}

Pour $|\tau| \leqslant N^{3 / 4}$, on a

$$
\zeta(s+\varepsilon)^{-1}-M_{N}(s+\varepsilon) \ll N^{-\varepsilon / 4}(1+|\tau|)^{1 / 2-\beta(\tau)},
$$

d'après la proposition 10, D'autre part,

$$
\begin{aligned}
|\zeta(s)|^{2} & \ll \exp (O(\log (3+|\tau|) / \log \log (3+|\tau|))) \\
& \ll(1+|\tau|)^{\beta(\tau)}
\end{aligned}
$$

donc

$$
\int_{|\tau| \leqslant N^{3 / 4}}|\zeta(s)|^{2}\left|\zeta(s+\varepsilon)-M_{N}(s+\varepsilon)\right|^{2} \frac{d \tau}{|s|^{2}} \ll N^{-\varepsilon / 2} \int_{-\infty}^{\infty}(1+|\tau|)^{-1-\beta(\tau)} d \tau,
$$

où la dernière intégrale est convergente.

Les deux propositions précédentes entraînent la proposition 3, ce qui achève la démonstration du théorème.

\section{Références}

[1] L. Báez-Duarte, On Beurling's real variable reformulation of the Riemann hypothesis, Adv. in Maths. 101 (1993), 10-30.

[2] L. Báez-Duarte, A class of invariant unitary operators, Adv. in Maths. 144 (1999), 1-12.

[3] L. Báez-Duarte, M. Balazard, B. Landreau et E. Saias, Notes sur la fonction $\zeta$ de Riemann, 3, Adv. in Maths. 149 (2000), 130-144.

[4] L. Báez-Duarte, A strengthening of the Nyman-Beurling criterion for the Riemann hypothesis, Rend. Mat. Acc. Lincei (9) 14 (2003), 5-11.

[5] M. Balazard et A. de Roton, Notes de lecture de l'article « Partial sums of the Möbius function » de Kannan Soundararajan, arXiv :0810.3587

[6] J.-F. Burnol, A lower bound in an approximation problem involving the zeroes of the Riemann zeta function, Adv. in Maths. 170 (2002), 56-70.

[7] J.-F. Burnol, On an analytic estimate in the theory of the Riemann zeta function and a theorem of Báez-Duarte, Acta Cientifica Venezolana 54 (2003), 210-215.

[8] H. Davenport, Multiplicative number theory, $3^{\text {rd }}$ edition revised by H.L. Montgomery, Springer, 2000.

[9] H.G. Diamond et K.S. McCurley, Constructive elementary estimates for $M(x)$, Analytic number theory, Lecture Notes in Mathematics 899, Springer (1981), 239-253.

[10] D.A. Goldston et S.M. Gonek, A note on $S(t)$ and the zeros of the Riemann zeta-function, Bull. London Math. Soc. 39 (2007), 482-486. 
[11] J.E. Littlewood, Quelques conséquences de l'hypothèse que la fonction $\zeta(s)$ de Riemann n'a pas de zéros dans le demi-plan $\Re s>\frac{1}{2}$, C.R.A.S. Paris 154 (1912), 263-266.

[12] H. Maier et H.L. Montgomery, The sum of the Möbius function, à paraitre au J. London Math. Soc.

[13] H.L. Montgomery, Ten lectures at the interface between analytic number theory and harmonic analysis, CBMS 84, AMS 1994.

[14] K. Soundararajan, Partial sums of the Möbius function, arXiv :0705.0723v2

[15] P. Tchebichef (sic), Mémoire sur les nombres premiers, J. Maths pures et appliquées, (Ser. I) 17 (1852), 366-390.

[16] E.C. Titchmarsh, The theory of the Riemann zeta-function, $2^{\text {nd }}$ edition revised by D.R. Heath-Brown, Oxford University Press, 1986.

[17] G. Tenenbaum, Introduction à la théorie analytique et probabiliste des nombres, $3^{\mathrm{e}}$ édition, Belin, 2008.

[18] E.T. Whittaker et G.N. Watson, A course of modern analysis, $4^{\text {th }}$ edition, Cambridge University Press, 1927.

BALAZARD, Michel

Institut de Mathématiques de Luminy, UMR 6206

CNRS, Université de la Méditerranée

Case 907

13288 Marseille Cedex 09

FRANCE

Adresse électronique : balazard@iml.univ-mrs.fr de ROTON, Anne

Institut Elie Cartan de Nancy, UMR 7502

Nancy-Université, CNRS, INRIA

BP 239

54506 Vandoeuvre-lès-Nancy Cedex

FRANCE

Adresse électronique : deroton@iecn.u-nancy.fr 